\title{
colaboraciones
}

\section{INSPECCION Y MANTENIMIENTO DE CHIMENEAS INDUSTRIALES}

\author{
(INSPECTION AND MAINTENANCE OF INDUSTRIAL CHIMNEYS)
}

\section{Fernando Pinilla Rodríguez,}

Ingeniero Técnico de Obras Públicas

\section{RESUMEN}

En este articulo se consideran brevemente diferentes aspectos relativos a la inspección de chimeneas.

Este trabajo intenta justificar la necesidad de una inspección periódica para evitar riesgos innecesarios.

Una chimenea que no sea inspeccionada con regularidad, puede llegar a ser un peligro para el área que la rodea y la reparación de sus daños puede alcanzar un coste muy elevado.
SUMMARY

In this article various aspects relating to the inspection of stacks are considered briefly.

This work intends to justify the necessity of a periodical inspection to avoid unnecessary risk.

A chimney without a regular inspection, can be a danger for the for surrounding area, and the repairing of its damage can reach a very high cost.

\section{INTRODUCCION}

Una chimenea es una estructura vertical por la que se conducen los gases procedentes de la combustión a una cierta altura.

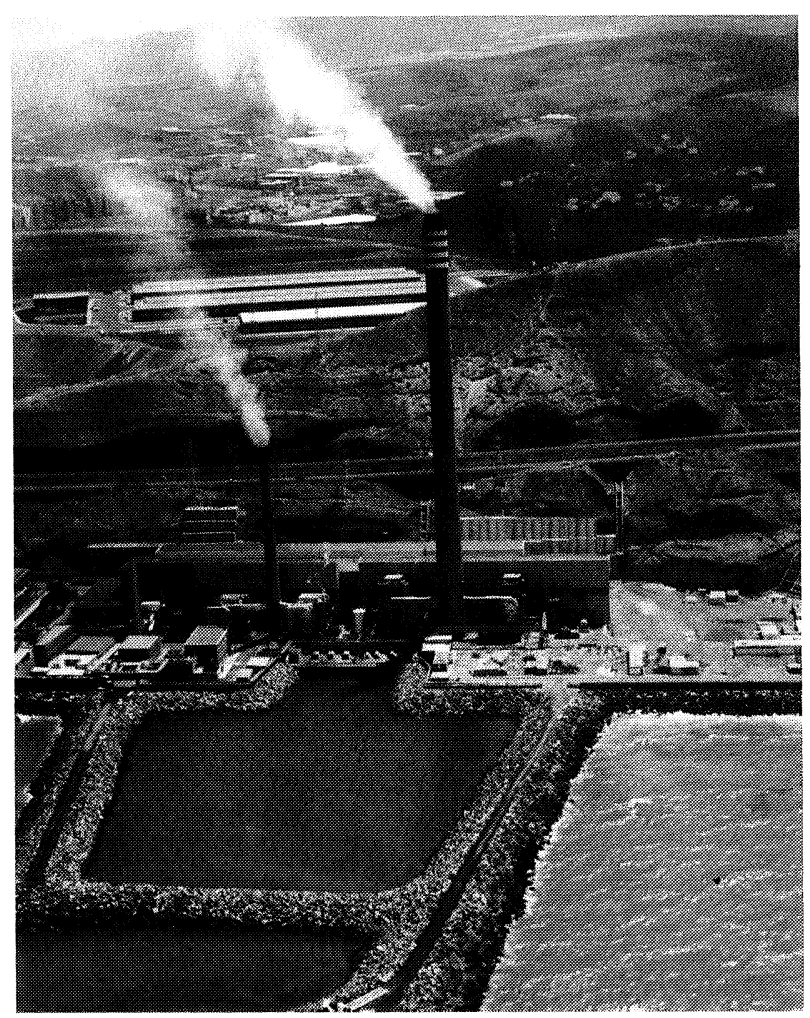

Sus partes principales son las siguientes:

- Cimentación.

- Fuste exterior.

- Conducto interior de gases.

- Accesorios: escaleras, plataformas, pararrayos, luces dẹ balizamiento, protección de la coronación, etc.

Las chimeneas se proyectan con o sin cámara de ventilación accesible entre el fuste exterior y el conducto de gases dependiendo del proceso de funcionamiento y de las posibilidades de inspección futura.

La elección de esta cámara para aislamiento y ventilación, depende principalmente de la temperatura a la que fluyen los gases por el conducto interior.

Los materiales utilizados en la construcción del conducto interior, dependen principalmente de la composición, temperatura y velocidad de los gases.

En la elección de los materiales con los que construir el fuste exterior influye, en primer lugar, la altura de la chimenea.

En una chimenea, como en cualquier otra instalación, se consideran tres clases de mantenimiento, cada uno con sus ventajas e inconvenientes:

- Mantenimiento correctivo: es el realizado como resultado del mal funcionamiento de la instalación. 


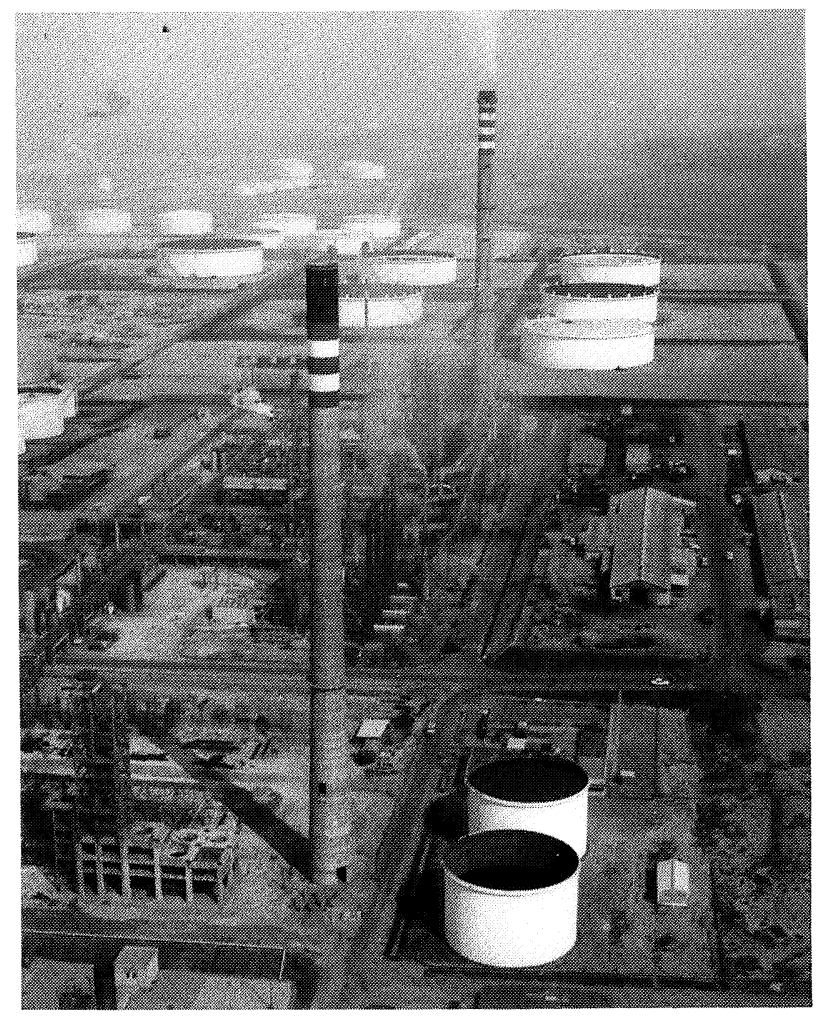

- Mantenimiento basado en la inspección: se realiza a consecuencia de una inspección previa.

- Mantenimiento preventivo: se efectúa conforme a un programa establecido de acuerdo con el funcionamiento de la fábrica.

\section{2. ¿POR QUE ES NECESARIA LA INSPECCION?}

El propósito de la inspección es determinar las condiciones de funcionamiento de la chimenea, tanto por el exterior como por el interior, con objeto de intentar predecir los deterioros que se producirán, así como las reparaciones necesarias a largo plazo.

Los resultados de una inspección proporcionan datos para el mantenimiento de la chimenea. Es necesario realizar con regularidad la inspección de una chimenea por las razones siguientes:

- Por seguridad para el área circundante.

- Para una emisión adecuada de gases por el conducto.

- Para detectar los daños en su fase inicial, y así evitar reparaciones más costosas.

- Para evitar paradas inesperadas de producción.

De la comparación entre el costo y los resultados de la inspección con el costo de mantenimiento, se puede deducir la frecuencia de un mantenimiento óptimo. En el apartado 9, «Frecuencia de la inspección», se desarro-
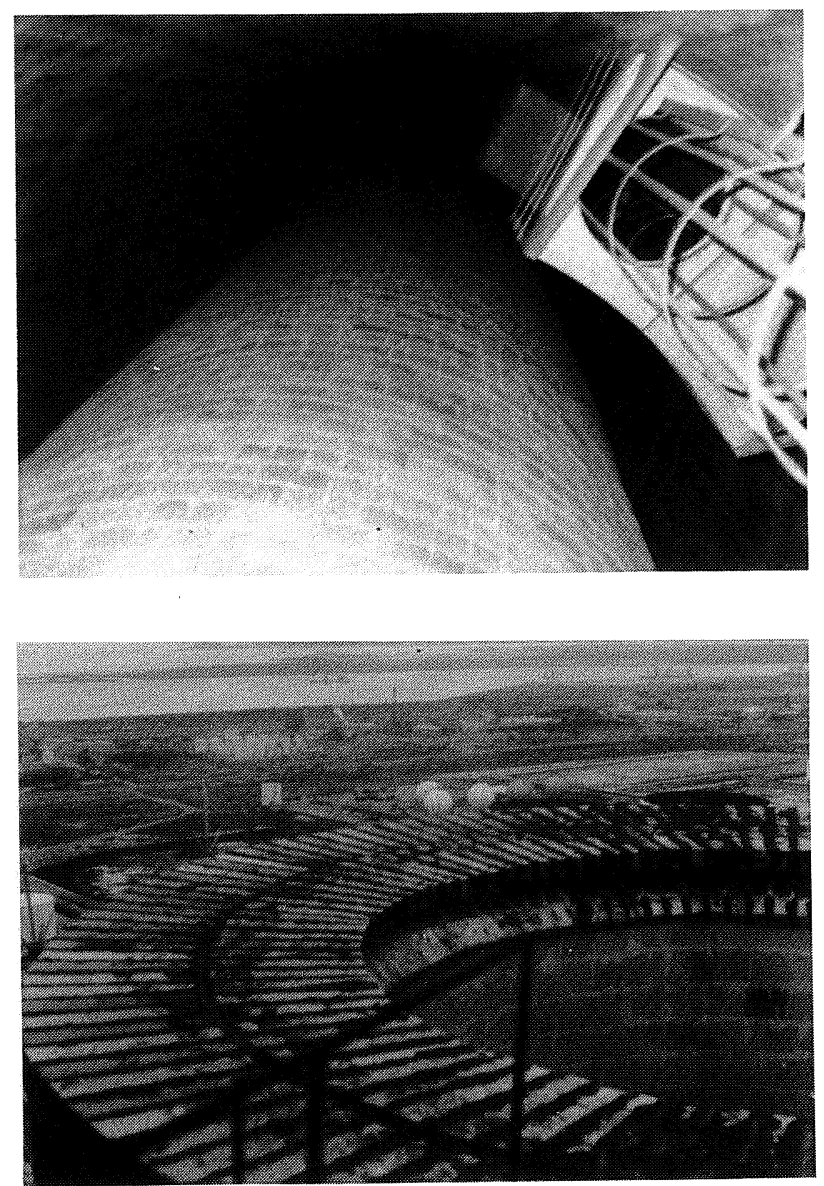

llará este punto más detenidamente. La realización de la inspección se basa en:

- Planos de proyecto y montaje.

- Libros de registro.

- Informes de reparaciones anteriores.

- Relación de puntos vulnerables.

- Lista de comprobaciones a realizar.

\section{3. ¿QUIEN DEBE REALIZAR LA INSPECCION?}

La inspección se deberá llevar a cabo por personal experimentado en supervisión de trabajos de este tipo y especialmente preparado para este fin.

Al ser las chimeneas estructuras altas y con mucha esbeltez, no pueden ser inspeccionadas por personas con problemas físicos, bien sea miedo a la altura o perturbaciones del equilibrio. Por ello los inspectores y supervisores deben someterse periódicamente a exámenes médicos.

Generalmente se recomienda que las inspecciones sean realizadas por el propio personal de la planta, sin embargo hay fábricas que las contratan a firmas independientes. En este caso es aconsejable hacerlo con una empresa especializada en la construcción de chimeneas, en que la inspección sea una parte de sus actividades. 

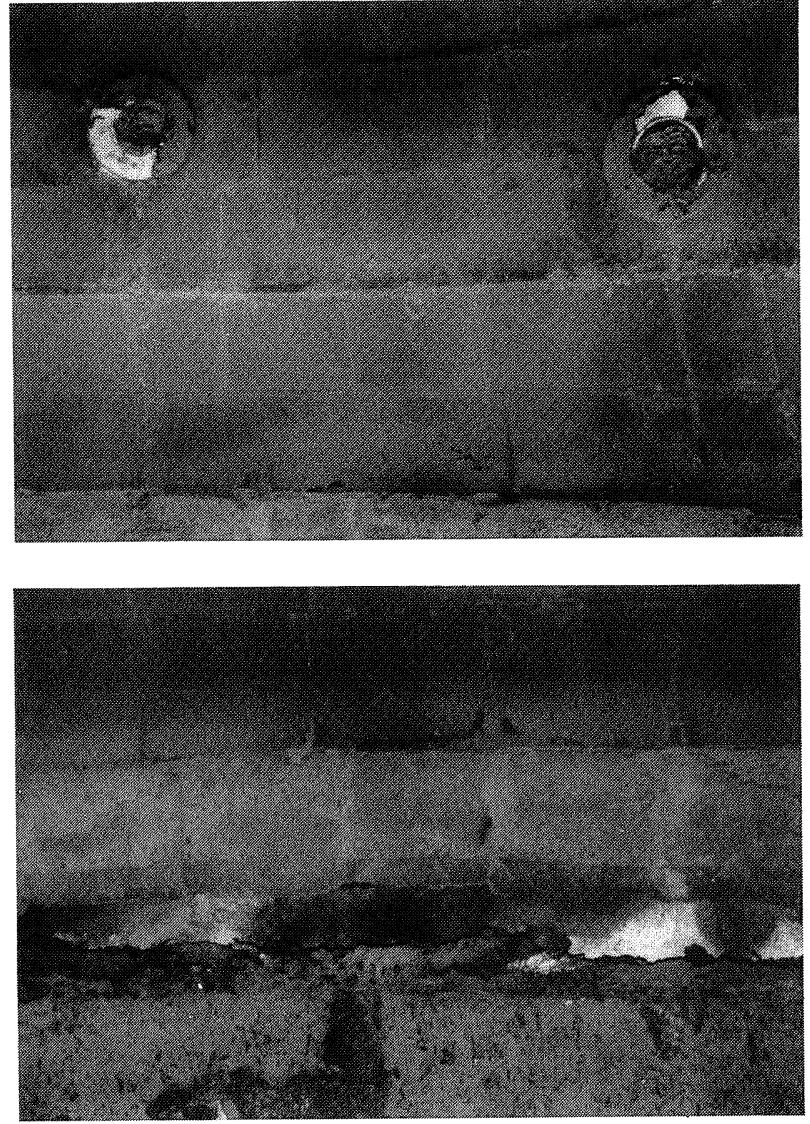

\section{4. ¿QUE DEBEMOS VER EN LA INSPECCION?}

Es importante conocer la situación y la magnitud de los daños que se pueden producir para decidir lo que se debe inspeccionar.

A continuación se relacionan las principales causas de deterioro en las diferentes partes de la chimenea.

\section{Exterior del fuste}

Causas:

- Climáticas: Influencia del viento. Variaciones de temperatura. Rayos de sol. Lluvia, nieve, hielo.

- Ambientales: Sustancias agrésivas en el aire procedente de instalaciones próximas.

- De proceso: Precipitación de gases sobre el fuste. Formación de condensados ácidos que atacan al fuste.

\section{Interior del fuste}

\section{Causas:}

- Climáticas: Lluvia, nieve.

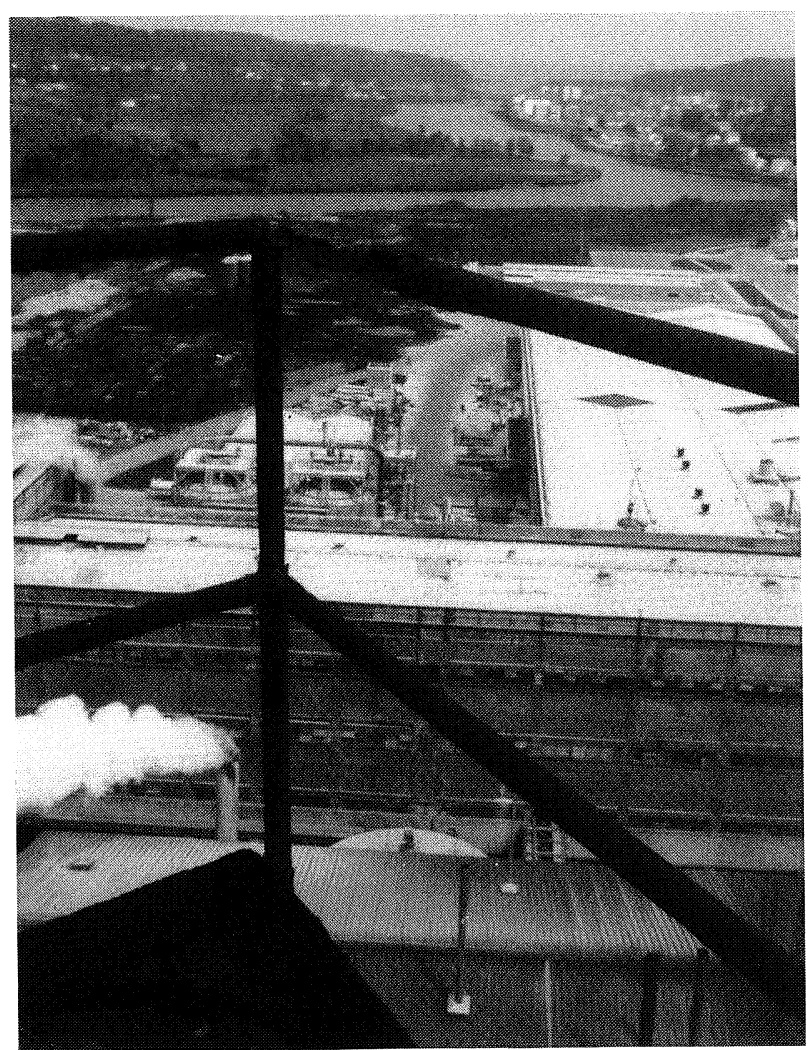

- De proceso (mecánicas): Cenizas volantes:

- Composición.

- Velocidad.

- Remolinos.

- De proceso (químicas): Gases:

- Composición.

- Velocidad.

- Humedad.

- Temperatura (punto de rocío).

- De proceso (físicas): Temperatura:

- Variación.

- Cambios bruscos.

\section{Accesorios}

Causas:

- Climáticas: Viento. Variaciones de temperatura. Rayos de sol. Lluvia, nieve, hielo.

- Ambientales: Sustancias agresivas en el aire procedentes de instalaciones próximas.

- De proceso: Precipitación de gases.

Una vez determinadas las causas y los daños que pueden producir las probables agresiones, se podrán dar una serie de recomendaciones para el debido mantenimiento. 

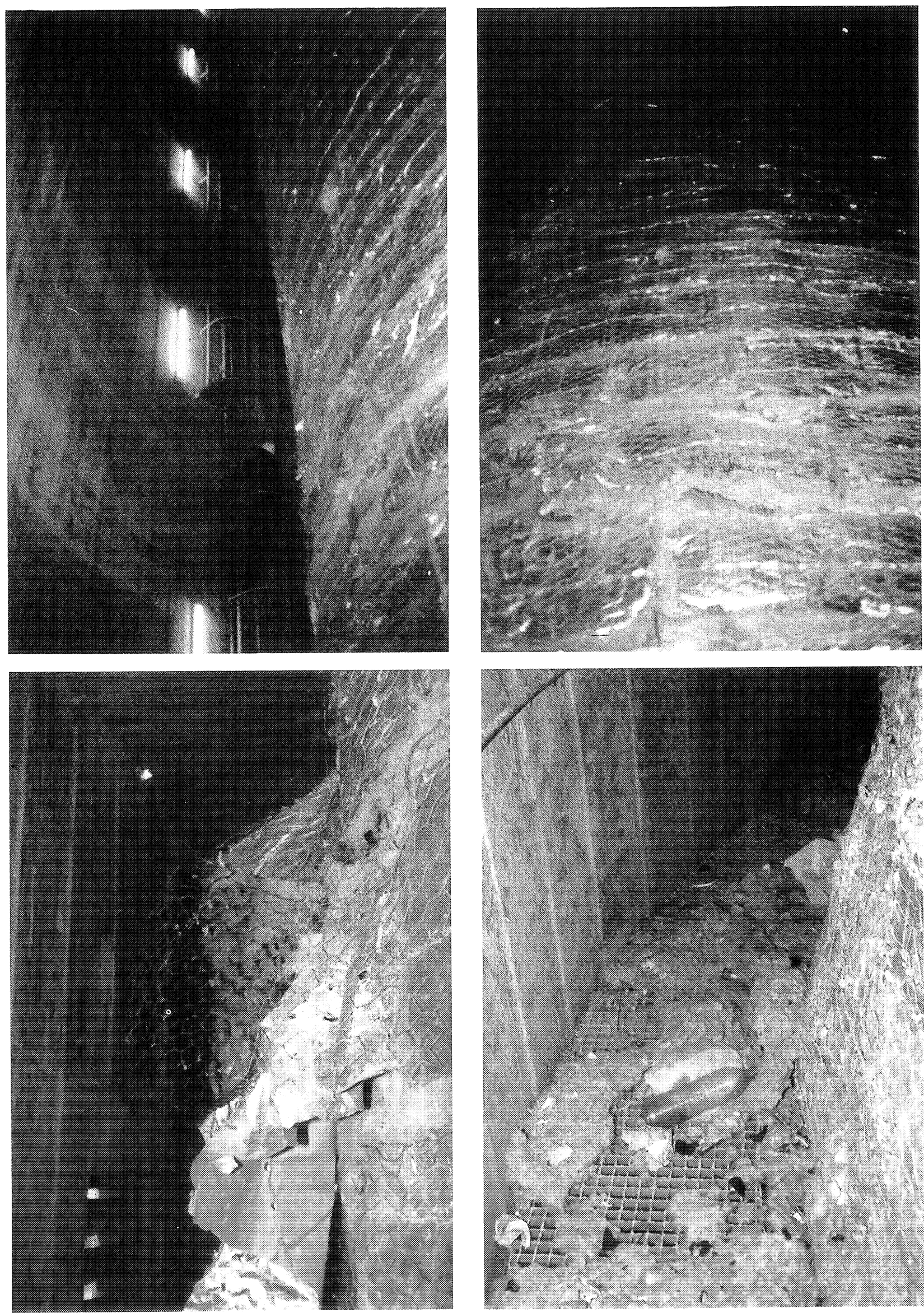
En la relación siguiente, se indican los desperfectos más comunes que pueden producirse en las chimeneas al cabo de un cierto tiempo de funcionamiento.

\section{Hormigón}

- Ataques producidos por la erosión.

- Formación de grietas y fisuras.

- Exfoliaciones (sonido hueco).

- Oxidación de las armaduras.

\section{Acero}

- Formación de óxido.

- Pérdida de espesor.

- Debilitamiento de las uniones:

- Soldaduras.

- Uniones atornilladas.

- Otros medios de unión.

\section{Ladrillos}

- Ataque producido por la erosión.

- Fisuras y grietas.

- Juntas:

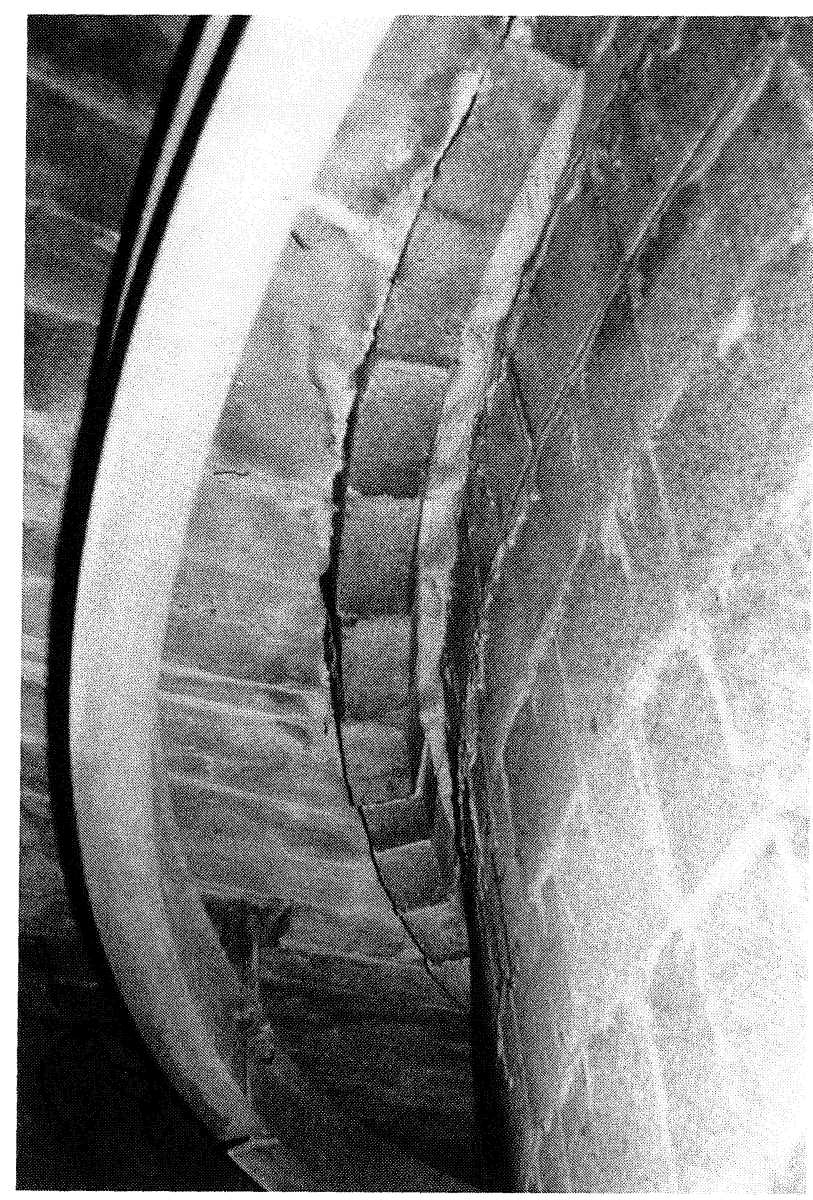

- Abiertas.

- Atacadas.

\section{- Daños en:}

- Juntas de expansión.

- Sellado.

- Anclajes.

- Bandas de fijación.

\section{Cámara de aire rellena con material aislante}

- Pérdida del relleno:

- Por deterioro del material de aislamiento.

- Por compactación del relleno.

- Ataque a la protección del paramento interior del fuste de hormigón.

- Mantas de fibras aislantes:

- Por deterioro del material.

- Roturas y grietas.

- Por pandeo o ahuecamiento.

- Debilidad en las costuras.

- Ataque a la protección del fuste de hormigón.

- Telas impermeables y a prueba de vapor:

- Roturas y grietas.

- Calidad del material y de las masillas.

- Solapes.

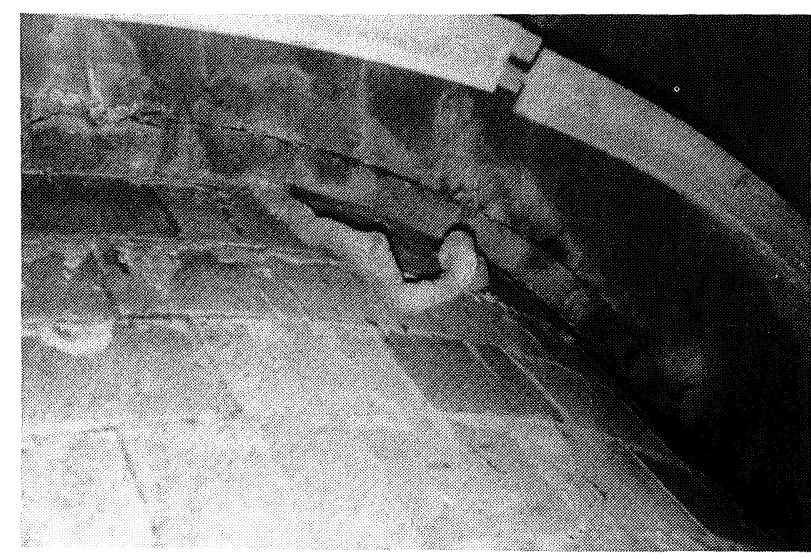

\section{Cámara de aire ventilada}

- Parcialmente cerrada como resultado de una circulación insuficiente.

- Aberturas de ventilación obstruidas.

- Débiles condiciones de protección del paramento interior del fuste de hormigón. 


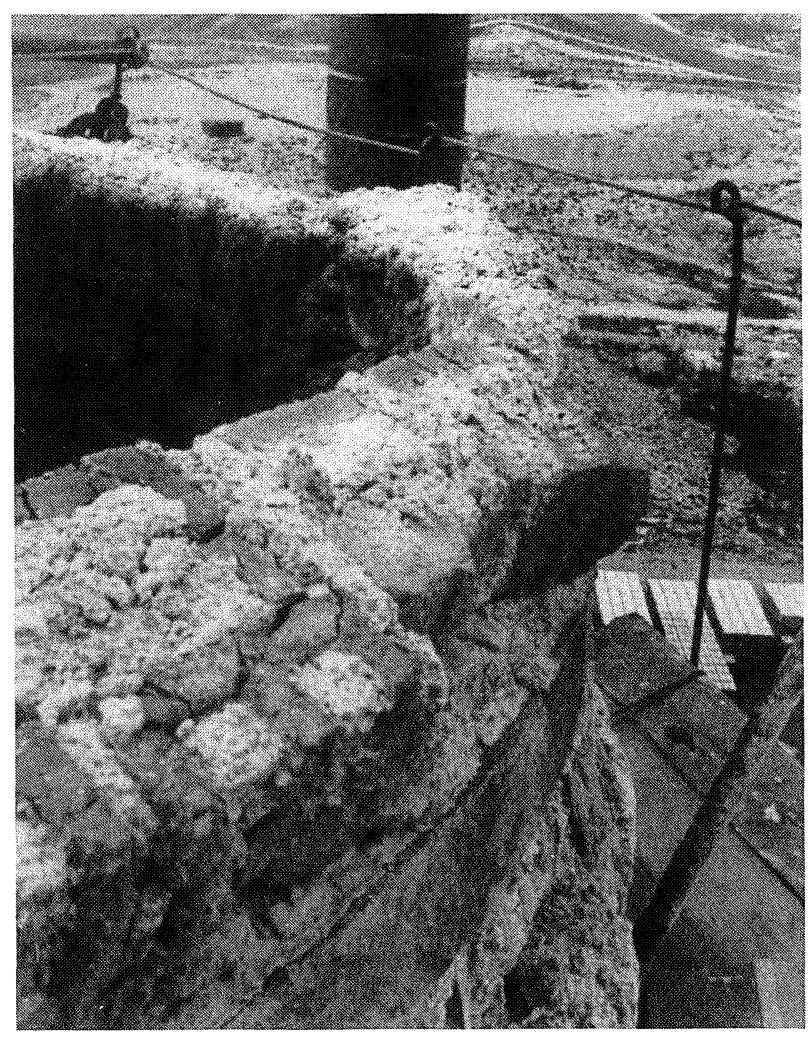

\section{Pintura de protección}

- Saponificación.

- Burbujas (ampollas), adherencias.

- Oxidación.

- Insuficiente espesor de la capa.

\section{Pararrayos}

- Estado de la punta.

- Conducción interrumpida.

- Toma de tierra insuficiente.

- Corrosión.

- Estado de los anclajes.

\section{Luces de balizamiento}

- Exceso de horas de las lámparas.

- Descenso de la intensidad luminosa.

- Cables de alimentación atacados.

- Filtraciones en las cajas de derivación.

— Estado de los anclajes y fijaciones.

Hay partes de la chimenea que requieren una atención

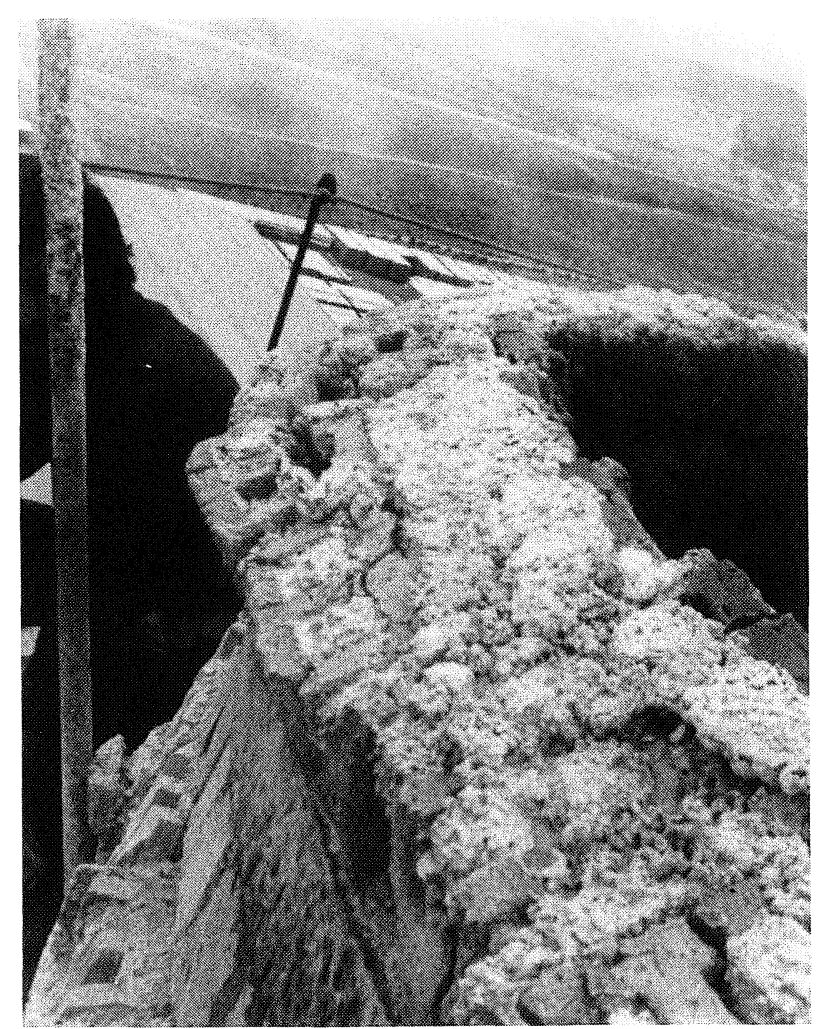

especial por ser las que tienen mayores posibilidades de ser atacadas. Estas son las siguientes:

En el exterior:

- Base.

- Exterior del fuste.

- Coronación: En chimeneas en funcionamiento la inspección puede ser dificultosa y a veces imposible, debido al peligro producido por la salida de los gases.

- Plataformas.

- Escaleras.

- Pararrayos: no olvidando la medición de su puesta a tierra.

- Luces de balizamiento.

Por el interior (con la chimenea parada):

- Entrada de gases.

- Interior del conducto de gases.

- Ménsulas.

- Placas de apoyo del revestimiento.

- Sellado de juntas.

- Juntas de dilatación

- Aislamiento e impermeabilización. 
Con la chimenea funcionando se puede medir la composición, temperatura y velocidad de los gases y cenizas volantes. Es posible hacerlo con ayuda de una sonda siempre que la chimenea esté provista de los orificios adecuados.

\section{METODOS DE ACCESO A LAS PARTES A INSPECCIONAR}

Dependiendo de la clase de inspección que se pretenda realizar, se puede acceder a las diferentes partes de la chimenea auxiliándose de los distintos medios que a continuación relacionamos.

- Elementos fijos instalados en la chimenea:

- Escaleras.

- Plataformas.

- Ascensores de cremallera por el exterior de la chimenea o por el interior de la cámara de aire.

- Equipos auxiliares instalados especialmente para ese fin, tales como:

- Cabrestantes con jaula para personas.

- Andamios motorizados.

- Andamios trepadores.

- Andamios tubulares montados desde el suelo.

El uso de estos equipos es muy costoso, no sólo por la maquinaria empleada sino también por el tiempo necesario para su utilización.

En general, los andamios tubulares instalados desde el suelo no se montan sólo para la inspección, sino que se utilizan cuando hay que realizar también simultáneamente una reparación.

Los otros medios sólo se utilizan cuando la inspección es completa, debido al alto costo que representa su uso (transporte, montaje, alquiler, etc.).

Para una inspección regular y periódica en las que el intervalo entre ellas no sea demasiado largo, será suficiente un examen desde las escaleras y plataformas, así como la medición de la toma de tierra del pararrayos. Siempre con la condición de completar la inspección en la próxima parada de la instalación.

\section{METODO DE INSPECCION}

Las observaciones pueden realizarse por medio de inspecciones visuales, mediciones y análisis o ensayos de laboratorio.

Puede ser una ayuda muy útil, llevar una pequeña grabadora durante la inspección, para así recordar cualquier cosa interesante que se observe.

También se recomienda hacer croquis de los daños que se observen aunque no son fáciles de hacer, por lo que se utilizan habitualmente cámaras fotográficas.
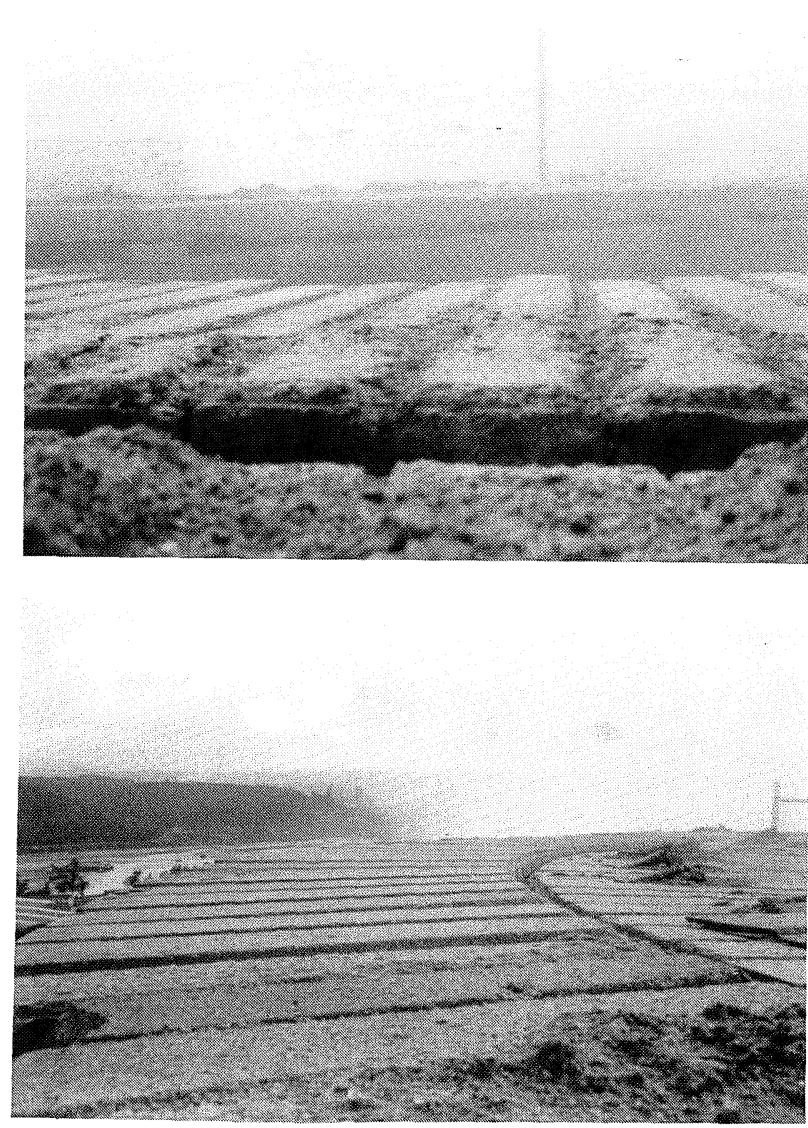

Cuando los daños deben ser juzgados por otros, son indispensables los textos, croquis y fotografías.

\section{Observaciones externas}

La inspección visual puede tener lugar en la proximidad de las escaleras, plataformas o andamios, pero en muchas ocasiones hay que observar zonas que están algo alejadas del punto más accesible.

En este caso se utilizan ayudas ópticas, tales como prismáticos, cámaras fotográficas dotadas con teleobjetivo y teodolitos. A veces se utiliza la combinación de rayo laser y teodolito y últimamente cámara de video. Para obtener una impresión general del estado de la chimenea, también se utiliza la técnica de la termografía (fotografias infrarrojas). Con ella se obtiene, con un alto grado de exactitud, la representación de las diferentes temperaturas en el exterior del fuste, dándonos una idea de la calidad del revestimiento, asi como del interior del conducto y su aislamiento.

\section{Observaciones internas}

La inspección visual del interior del conducto normalmente se realiza aprovechando el tiempo durante el cual la chimenea permanece fuera de servicio debido a una parada de la instalación.

Las chimeneas con cámara de aire accesible pueden inspeccionarse durante su funcionamiento siempre que estén provistas de agujeros adecuados. 

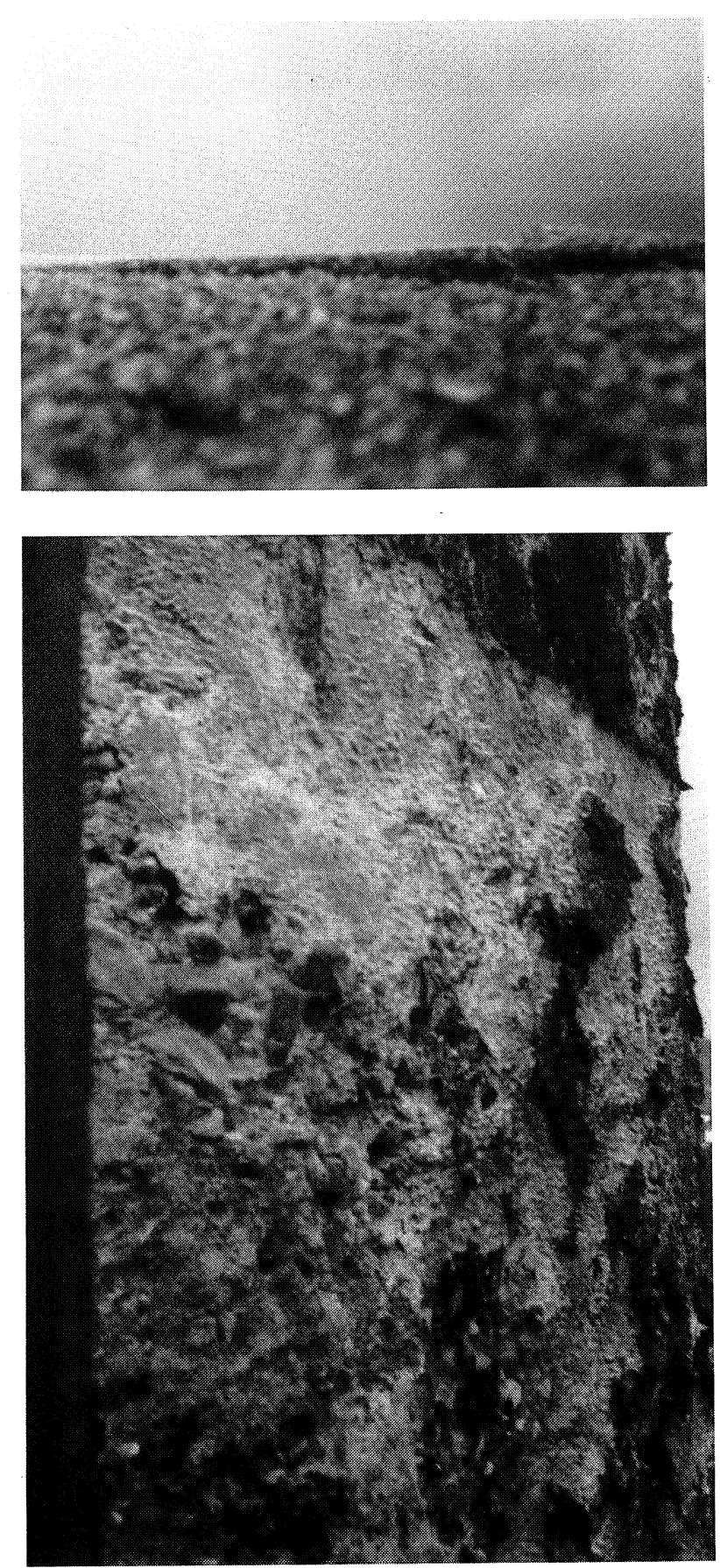

En casos especiales también es posible realizar la inspección durante el funcionamiento de la chimenea, utilizando las siguientes técnicas:

- Fotografias infrarrojas: Para la determinación de fisuras y partes aplastadas en el revestimiento cerámico.

- Video: Utilizando cámaras resistentes a la temperatura. Actualmente existen cámaras que resisten temperaturas de hasta $250{ }^{\circ} \mathrm{C}$.

- Exámenes destructivos: Extracción de testigos por perforación.
Dependiendo de la naturaleza y composición de las cenizas volantes, se pueden extraer parte de los depósitos sobre el conducto para ser examinados posteriormente en el laboratorio. No debe utilizarse agua para esta operación por las reacciones químicas que se pueden producir.

Se debe prestar mucha atención a las zonas situadas detrás del conducto, porque a veces, aún estando el interior en buenas condiciones, estas zonas pueden haber sido atacadas en mayor o menor grado. Las principales son:

- Placas soporte del conducto y ménsulas de apoyo de las placas.

Como resultado de una pérdida de espesor de conducto, se produce un aumento de temperatura en el hormigón. También en esa zona se desprende el aislamiento con más facilidad, debido a lo irregular de su superficie.

\section{- Aislamiento.}

Especialmente cuando es a base de vermiculita o perlita al ser sensibles a la humedad.

- Protección de la superficie interior del fuste de hormigón.

Se producen condensaciones como resultado de la diferencia de temperatura entre la capa exterior del fuste, relativamente fría y la caliente del interior del conducto. Se produce una reacción química dando como resultado la formación de ácidos.

Para facilitar la inspección de estas zonas mencionadas, es necesario remover algunos ladrillos. Dependiendo de la altura de la chimenea se extraerán en varios puntos, todos en el tercio superior que es el más sensible al ataque ácido. Se localizarán debajo de las ménsulas y placas soporte del conducto, así como donde se localicen fugas de gases por juntas o grietas. Asimismo en todos los puntos en donde se hayan producido o puedan producirse daños.

\section{MEDICIONES}

Las mediciones son un complemento indispensable de las inspecciones. La mayoría de ellas pueden ser realizadas directamente por el inspector.

A continuación se describen las mediciones más comunes que deben realizarse en una inspección, así como los instrumentos que se utilizan normalmente:

- Teodolito: Desviaciones del fuste exterior.

- Esclerómetro: Resistencia del hormigón medida por aplastamiento.

- Valor pH: Por medio de fluidos indicadores. Los valores deben ser superiores a 9,5 para proteger las armaduras de la corrosión. 
- Bandas elásticas: Por medio de bandas elásticas pegadas sobre las grietas, es posible determinar si los daños son activos o se han estabilizado.

- Termopares: Medidores de temperatura del conducto, bien en el interior del revestimiento o en la corriente de gases.

- Ensayos de laboratorio: Deterioro físico-químico de los materiales de construcción. Composición de cenizas volantes y de los gases.

- Micrómetro: Medidas de espesor de la protección aplicada como pintura.

- Equipo de ultrasonidos: Espesor de conductos metálicos.

\section{TOMA DE MUESTRAS}

La toma de muestras tiene por objeto la determinación de la naturaleza y grado de ataque de los materiales por medio de testigos físicos y químicos.

Normalmente se utilizan taladros para la extracción de los testigos. Estos, de 25 a $100 \mathrm{~mm}$ de diámetro, se perforan con coronas de diamante. Los taladros deben ir dotados del agua necesaria (depósitos y bomba).

No se aconseja utilizar equipos neumáticos de perforación.

Algunas chimeneas están equipadas con orificios para toma de muestras. En ellas el muestreo puede realizarse en cualquier momento, con la chimenea en funcionamiento o fuera de servicio.

\section{FRECUENCIA DE LA INSPECCION}

Depende de varios factores, tales como:

- Situación de la chimenea.

- Tipo de construcción (hormigón, ladrillo, acero, materiales sintéticos, etc.).

- Estado de conservación.

- Programa de mantenimiento.

A partir de la inspección se debe determinar la extensión exacta de los daños para la realización de los trabajos de reparación.

Se utilizan para la reparación los mismos andamios o cabrestantes provistos de jaula, utilizados para la inspección.

Como existen diferencias entre las inspecciones del fuste y del conducto, a continuación ambas se desarrollan por separado.

\section{Fuste y accesorios}

En general se recomiendan ciclos de inspección no demasiado largos.

Se puede programar un periodo de tres años:

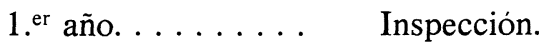

$2 .^{\circ}$ año........ Reparación.

$3{ }^{\text {er }}$ año. . . . . S S in actividad.

Hay compañias que han optado por realizar anualmente una inspección visual más o menos superficial desde las escaleras y plataformas.

La minuciosidad de la inspección dependerá del estado de conservación de la chimenea, influencia de la antigüedad del proceso, programa de mantenimiento y periodo de tiempo que la chimenea permanece en funcionamiento.

Se recomienda no superar el ciclo de tres años con el fin de prevenir problemas inesperados, sobre todo relacionados con la seguridad.

La ejecución de los trabajos se debería realizar preferentemente durante los meses de mayo a septiembre, debido a las mejores condiciones meteorológicas.

\section{Conducto interior}

La posibilidad de inspección del conducto interior depende de los siguientes factores:

- Cámara de aire (accesible o no).

- Equipos auxiliares propios de la chimenea (escotillas, agujeros toma de muestras).

- Proceso de funcionamiento continuo o discontinuo.

No es aconsejable dejar la chimenea fuera de funcionamiento solamente para realizar la inspección, para no alterar el proceso. El enfriamiento puede causar serios daños en el revestimiento. El ataque tiene lugar especialmente dentro de la primera semana posterior al enfriamiento.

En la práctica se ha demostrado que en chimeneas de operación continua sin gases agresivos, como instalaciones de alta temperatura o baterias de cok, el estado de los conductos puede ser muy aceptable después de largo tiempo de funcionamiento ( 25 años o más).

En suma, se puede establecer que:

- Se deben planificar las paradas para un funcionamiento óptimo de la chimenea.

- Siempre que sea posible, se deben instalar equipos auxiliares en la chimenea con objeto de permitir inspecciones sin necesidad de parar la instalación. 